\title{
考虑附加强化效应的多轴高周疲劳损伤演化模型*
}

\author{
彭 艳 ${ }^{1,2}$ 李浩然 ${ }^{1}$ \\ (1. 燕山大学国家冷轧板带装备及工艺工程技术研究中心 秦皇岛 066004; \\ 2. 燕山大学亚稳材料制备技术及科学国家重点实验室 秦皇岛 066004)
}

\begin{abstract}
摘要: 基于连续介质损伤力学理论并结合修正循环强度系数法, 研究非比例加载对材料高周疲劳寿命的影响, 建立一种多轴 应力当量折算高周疲劳损伤强化模型。同时根据常规微塑性应变高周疲劳损伤演化模型给出的 $S-N$ 曲线方程以及修正循环强 度系数法基本原理对所建模型的材质参数识别方法进行阐述。以航空工业常用的金属材料铝合金 $\mathrm{LY} 12 \mathrm{CZ}$ 和 $30 \mathrm{CrMnSiA}$ 钢 为计算实例, 得到两种材料的模型参数。将已建的损伤演化模型嵌入到 ABAQUS 的 UMAT 子程序中, 实现了对受损试件的 损伤追踪以及寿命预测。两种材料计算结果表明, 新建模型的预测效果均在 3 倍误差以内, 与试验吻合较好。此外, 还对比 分析现有的其他多轴寿命预测模型的预测效果, 结果表明, 新建模型更适合于多轴高周瘦劳寿命预测。
\end{abstract}

关键词: 损伤力学; 多轴高周疲劳; 非比例加载; 参数识别; 当量折算

中图分类号: $\mathrm{O} 346$

\section{Multiaxial High Cycle Fatigue Damage Evolution Model Including Additional Hardening Effect}

\author{
PENG Yan ${ }^{1,2}$ LI Haoran ${ }^{1}$ \\ (1. National Engineering Research Center for Equipment and Technology of Cold Strip Rolling, \\ Yanshan University, Qinhuangdao 066004; \\ 2. State Key Laboratory of Metastable Material Science and Technology, \\ Yanshan University, Qinhuangdao 066004)
}

\begin{abstract}
Based on continuum damage mechanics theory and corrected cycle intensity factor method, the effect of non-proportional loading on high-cycle fatigue life is investigated. Consequently, a hardening model with multiaxial stress equivalent conversion and high cycle fatigue damage is established. Simultaneously, in the base of $S-N$ curve equation based on the conventional micro-plastic strain high-cycle fatigue damage evolution model and the basic principles of corrected cycle intensity factor method, a material parameter identification method of model is described. Take the metal material of aluminum alloy LY12CZ and 30CrMnSiA steel commonly used in aeronautic industry as examples, model parameters of two kinds of material are obtained. Embed the established damage evolution model into the UMAT subroutine in ABAQUS, and realize the damage tracking and life prediction for specimens. The calculation results of two materials show that the predictive effect of new models are both in the error of less than 3 times, and in good agreement with experiment. In addition, compare and analyze the predictive effect of other existing prediction models, and the results indicate that the new developed model is more suitable for life prediction of multiaxial high cycle fatigue.
\end{abstract}

Key words: damage mechanics; multiaxial high-cycle fatigue; non-proportional loading; parameters identification; equivalent conversion

\section{0 前言}

疲劳是指材料在往复载荷作用下发生损伤失 效的一种形式。据不完全统计, 在现代工业领域,

*国家科技支撑计划资助项目(2011BAF15B01)。20140909 收到初稿, 20141211 收到修改稿
约有 $80 \%$ 的构件破坏都是由于疲劳失效引起的 ${ }^{[1]}$ 。 疲劳失效成为制约延长构件使用寿命的主要因素之 一, 同时疲劳损伤分析也成为学术界以及工程领域 比较关注的一类问题。早期研究疲劳问题依赖于试 验且加载形式较为简单, 基本为单轴加载, 利用一 定量的试验数据并结合统计方法确定材料的疲劳损 伤规律。而现代构件一般受载较为复杂, 单轴疲劳 
寿命预测理论已远远不能满足人们的抗疲劳设计, 多轴疲劳寿命预测理论伴随着电液伺服疲劳试演机 的投入使用取得了较快发展。代表性主要有应力不 变量法、临界面法以及细观积分法等 ${ }^{[2-4]}$ 。 CROSSLAND 等 ${ }^{[5-6]}$ 分别以应力第一不变量和应力 偏张量的第二不变量作为损伤控制参数构建了多轴 疲劳失效准则, 但是这两种准则应用于多轴非比例 载荷下的预测效果普遍偏于非保守 ${ }^{[7]}$ 。金丹等 ${ }^{[8]}$ 以 Mod. $9 \mathrm{Cr}-1 \mathrm{Mo}$ 钢缺口试样作为试验材料进行了多种 加载路径下的多轴疲劳试验, 并采用临界面法进行 了寿命预测, 预测结果较为理想。虽然临界面法在 多轴疲劳寿命预测中应用较为广泛, 但是如何选取 临界面依然存在争议 ${ }^{[9]}$ 。

近年来, 作为一种被公认为非常有前途的研究 方法一一损伤力学法的发展较快 ${ }^{[10]}$, 损伤力学法依 据热力学基本原理推演损伤演化方程, 具有确定的 物理和力学意义。有些传统的疲劳寿命预测理论在 损伤力学的理论框架下得到了合理的推演, 揭示了 其力学意义 ${ }^{[11]}$ 。对于高周疲劳损伤, 损伤力学通过 引入细观微塑性应变建立了微塑性应变高周疲劳损 伤演化模型, 模型克服了 Miner 线性损伤累积的不 足之处, 体现了损伤的非线性累积, 这与试验结果 基本符合。然而对于非比例多轴高周疲劳而言, 由 于应力应变主轴的不断旋转造成材料的多滑移系被 开动, 使得材料相对于比例加载或单轴加载有着更 快的损伤累积和更短的疲劳寿命 ${ }^{[12]}$ 。

本文基于损伤力学理论并考虑非比例加载历 史对材料疲劳寿命的影响, 引入修正微塑性循环强 度系数法, 以期能更好地描述材料在非比例载荷作 用下的损伤演化历程。另外, 本文结合常规微塑性 应变高周疲劳模型给出的单轴对称拉压寿命预测值 以及修正循环强度系数法基本原理, 给出伤演模型 材料参数识别方法。最后, 选取两种不同类型材料 的多轴高周疲劳试验数据, 利用 ABAQUS 有限元 软件计算两种材料在不同加载路径下的疲劳寿命, 分析新建模型的预测效果。

\section{1 多轴高周疲劳损伤演化方程}

\section{1 连续介质损伤力学基本理论}

疲劳损伤作为一种不可逆过程, 在热力学框架 下需要满足热力学第二定律, 即 Clausius-Duhamel 不等式表述如下 ${ }^{[13]}$

$$
\dot{\psi}_{\mathrm{p}}-Y \dot{D}-T^{-1} \boldsymbol{\Gamma} \dot{\boldsymbol{q}}>0
$$

式中, $\dot{\psi}_{\mathrm{p}}$ 表示塑性变形热耗散功率; $-Y$ 为广义损 伤驱动力; $\dot{D}$ 为损伤演化率; $T$ 表示热力学温度; $\boldsymbol{\Gamma}$
表示温度递度矢量； $\dot{\boldsymbol{q}}$ 表示热流密度矢量。当假定 塑性耗散与损伤耗散不耦合以及疲劳损伤被认为是 一种缓慢的等温过程时，式(1)可简述为 ${ }^{[14]}$

$$
-Y \dot{D}>0
$$

文献[13]中定义 $-Y$ 为广义损伤驱动力, 具体表述为

$$
\begin{gathered}
-Y=\frac{\sigma_{\mathrm{eq}}^{2} R_{\mathrm{v}}}{2 E(1-D)^{2}} \\
R_{\mathrm{v}}=2(1+\nu) / 3+3(1-2 \nu)\left(\sigma_{\mathrm{m}} / \sigma_{\mathrm{eq}}\right)^{2}
\end{gathered}
$$

式中, $D$ 为损伤变量，当 $D$ 到达临界值 $D_{\mathrm{c}}$ 时，材 料破坏; $E 、 \nu$ 分别为材料的弹性模量和泊松比; $R_{v}$ 为三轴应力比, 当构件处于单向拉压状态, $R_{v}=1$; $\sigma_{\mathrm{eq}}$ 为 von Mises 等效应力; $\sigma_{\mathrm{m}}$ 为静水应力。考虑 到式(3)的非负性，满足式(2)的基本条件为

$$
\dot{D}>0
$$

这表明当满足 Clausius-Duhamel 不等式(1)时，损伤 变量具有递增性。对于高周疲劳而言, 损伤变量 $D$ 演化方程的 $t$ 型描述由 LEMAITRE一般意义上损伤 演化方程表述为 ${ }^{[15]}$

$$
\dot{D}=\left(\frac{-Y}{S_{1}}\right)^{S_{2}} \dot{e}_{\mathrm{p}}
$$

式中, $S_{1} 、 S_{2}$ 均为材料常数, 由于高周疲劳损伤非 线性较弱, $S_{2}=1^{[16]}$; $\dot{e}_{\mathrm{p}}$ 为累积等效微塑性应变率。 文献[15]假定微塑性累积应变量 $e_{\mathrm{p}}$ 满足 RambergOsgood 关系式，即

$$
e_{\mathrm{p}}=\left[\frac{\sigma_{\mathrm{eq}}}{K(1-D)}\right]^{\frac{1}{n}}
$$

此时一维单轴拉拉高周疲劳损伤本构方程为

$$
\mathrm{d} D=\frac{P \sigma^{Q}}{(1-D)^{Q+1}} \mathrm{~d} \sigma
$$

式中, $\sigma$ 为加载应力; $P, Q$ 均为材料常数, 两者的 展开形式为

$$
\left\{\begin{array}{c}
P=\frac{K^{-\frac{1}{n}}}{2 n E S_{1}} \\
Q=1+\frac{1}{n}
\end{array}\right.
$$

式中, $K$ 为材料的微塑性循环强度系数; $n$ 为微塑 性循环应变硬化指数。由损伤本构方程式(7)以及损 伤变量 $D$ 的递增性可知, 只有当加载时, 才会引入 损伤。通过单次损伤累积积分计算可将式(7)改写成 $N$ 型描述为 


$$
\frac{\mathrm{d} D}{\mathrm{~d} N}=\frac{P}{(Q+1)(1-D)^{Q+1}}\left(\sigma_{\max }^{Q+1}-\sigma_{\min }^{Q+1}\right)
$$

式中, $N$ 为循环周次; $\sigma_{\text {max }}$ 为加载段最大应力值; $\sigma_{\min }$ 为加载段最小应力值。

\section{2 计及附加强化效应的多轴疲劳损伤演化模型}

文献[14]指出 LEMAITRE 在试验中发现, 材料 的弹性模量随着损伤的发展而不断降低, 认为损伤 与材料的弹性有关, 即 Helmholtz 自由能弹性部分 与损伤变量耦合, 塑性部分不反映损伤, 于是

$$
W_{\mathrm{e}}=W_{\mathrm{e}}(D, T)
$$

式中, $W_{\mathrm{e}}$ 表示材料弹性应变能密度; 特殊的, 当 $D=0$ 时, 表示无损状态下弹性应变能密度。将式(10) 按多元函数泰勒级数展开并忽略高阶微量可得

$$
W_{\mathrm{e}}^{(N+1)}=W_{\mathrm{e}}^{(N)}+\frac{\partial W_{\mathrm{e}}}{\partial D}\left(D_{N+1}-D_{N}\right)+\frac{\partial W_{\mathrm{e}}}{\partial T}\left(T_{N+1}-T_{N}\right)
$$

式中, $W_{\mathrm{e}}^{(N)} 、 W_{\mathrm{e}}^{(N+1)}$ 分别表示当前应力状态下材料 的弹性应变能密度和下个加载周期中与当前时刻相 差整周期的弹性应变能密度; $T_{N} 、 T_{N+1}$ 分别表示当 前材料温度和下个加载周期中与当前时刻相差整周 期的材料温度; $D_{N} 、 D_{N+1}$ 分别表示当前时刻损伤 变量和下个加载周期的损伤变量。为了得到式(11) 具体的展开形式, 可将损伤变量 $D$ 按循环周次 $N$ 泰 勒级数展开并忽略高阶微量可得

$$
D_{N+1}=D_{N}+\frac{\mathrm{d} D}{\mathrm{~d} N} \times 1
$$

当假定损伤过程是一等温过程, 由式(12)可将式(11) 改写为

$$
W_{\mathrm{e}}^{(N+1)}=W_{\mathrm{e}}^{(N)}+\frac{\partial W_{\mathrm{e}}}{\partial D} \frac{\mathrm{d} D}{\mathrm{~d} N}
$$

式(13)表明如果多轴应力状态下的弹性应变能密度 与某一单轴应力状态下的弹性应变能密度在任意时 刻均相等, 那么损伤演化动率 $\mathrm{d} D / \mathrm{d} N$ 完全相等, 即 损伤历程完全等效。基于此, 可将多轴应力折算为 单向应力状态并计及压缩应力对裂纹的闭合效应, 即 $^{[17]}$

$$
\sigma= \begin{cases}\sigma_{\text {eq }} \sqrt{R_{v}} & \left|\sigma_{1}\right| \geqslant\left|\sigma_{3}\right| \\ \left(\frac{1-D}{1-h D} R_{v}\right)^{\frac{1}{2}} \sigma_{\text {eq }} & \left|\sigma_{1}\right|<\left|\sigma_{3}\right|\end{cases}
$$

式中, $h$ 为裂纹闭合因子, LEMAITRE 建议 $h=0.2$; $\sigma_{1}, \sigma_{3}$ 分别为第一和第三主应力。

考虑到一个折算载荷循环中可能存在多个加 载分段, 故而当不考虑非比例附加强化效应时, 将
单轴拉拉疲劳损伤演化方程式(9)推广到多轴应力 状态并写成分段表述为

$$
\frac{\mathrm{d} D}{\mathrm{~d} N}=\frac{P}{(Q+1)(1-D)^{Q+1}} \sum_{i=1}^{m}\left(\sigma_{\mathrm{i}, \max }^{Q+1}-\sigma_{\mathrm{i}, \min }^{Q+1}\right)
$$

式中, $m$ 表示加载分段数目; $\sigma_{\mathrm{i}, \max }$ 和 $\sigma_{\mathrm{i}, \text { min }}$ 分别为 折算载荷谱中加载分段所对应的峰谷点应力值。

对于非比例多轴高周疲劳而言, 由于应力应变 主轴的不断旋转造成材料的潜在滑移系被开动, 滑 移系之间的交互作用使得材料呈现附加强化效应。 为此, 本文引入旋转因子参量 $F$, 予以表征材料的 潜在滑移系的开启度。旋转因子参量 $F$ 的具体表述 形式为

$$
F=\frac{\Delta \tau_{\min }}{\Delta \tau_{\max }}
$$

式中, $\Delta \tau_{\min }$ 表示加载历程中的最小切应力幅; $\Delta \tau_{\text {max }}$ 表示加载历程中的最大切应力幅。考虑到比 例加载时主应力平面不变性, $F=0$; 非比例加载 时, 由于存在强化效应, $F \neq 0$ 。

对于无平均应力保持正弦波加载下的扭拉光 滑薄壁圆管试件, 其 $F$ 值经推演可得

$$
F=\sqrt{\frac{4 \lambda_{\sigma}^{2}+1-\sqrt{\left(4 \lambda_{\sigma}^{2}-1\right)^{2}+16 \lambda_{\sigma}^{2} \cos ^{2} \delta}}{4 \lambda_{\sigma}^{2}+1+\sqrt{\left(4 \lambda_{\sigma}^{2}-1\right)^{2}+16 \lambda_{\sigma}^{2} \cos ^{2} \delta}}}
$$

式中, $\lambda_{\sigma}$ 为扭拉应力幅值比, $\delta$ 为扭拉加载相位 差值。

由于材料的本构方程与其位错滑移组态密切 相关, 为了将材料细微观组织结构变化反映到宏观 物理量上, 建立联系宏细微观的微塑性本构方程, 本文依据修正循环强度系数法构建多轴微塑性本构 关系。修正循环强度系数法, 顾名思义, 即通过修 正循环强度系数来考虑非比例加载下的附加强化并 忽略循环应变硬化指数 $n$ 的变化 ${ }^{[18]}$ 。此时, 微塑性 循环强度系数 $K$ 经修正为

$$
K_{\text {nonp }}=(1+g F) K
$$

式中, $K_{\text {nonp }}$ 表示非比例加载路径下的微塑性循环强 度系数; $g$ 为材料常数, 表征多轴非比例附加强化 效应也取决于材料本身。将式(18)代入式(5)中并计 拉压异性, 可得多轴疲劳损伤本构方程为

$$
\mathrm{d} D= \begin{cases}\frac{(1+g F)^{-\frac{1}{n}} P R_{\mathrm{v}}^{S_{1}} \sigma_{\mathrm{eq}}^{Q}}{(1-D)^{Q+1}} \mathrm{~d} \sigma_{\text {eq }} & \left|\sigma_{1}\right| \geqslant\left|\sigma_{3}\right| \\ \frac{(1+g F)^{-\frac{1}{n}} P h^{S_{1}} R_{\mathrm{v}}^{S_{1}} \sigma_{\text {eq }}^{Q}}{(1-h D)^{Q+1}} \mathrm{~d} \sigma_{\text {eq }} & \left|\sigma_{1}\right|<\left|\sigma_{3}\right|\end{cases}
$$


由前面推演可知, 当不考虑附加强化效应时, 基于式(18)的伤演模型 $N$ 型描述已做等效折算处 理, 已由式(15)给出。考虑到对于某一确定的加载 路径而言, $F$ 为某一常量, 因此由式(19)可导出计 及非比例附加强化效应的多轴高周疲劳损伤演化方 程, 即

$$
\frac{\mathrm{d} D}{\mathrm{~d} N}=\frac{P(1+g F)^{-\frac{1}{n}}}{(Q+1)(1-D)^{Q+1}} \sum_{\mathrm{i}=1}^{m}\left(\sigma_{\mathrm{i}, \max }^{Q+1}-\sigma_{\mathrm{i}, \text { min }}^{Q+1}\right)
$$

\section{2 伤演模型材料参数识别方法}

基于微塑性高周疲劳损伤演化模型式(5)并计 及拉压异性可得构件在对称往复载荷下疲劳寿命预 测值为 ${ }^{[19]}$

$$
N_{\mathrm{f}}=\Phi \sigma_{\mathrm{a}}^{-(Q+1)}
$$

写成双对数形式为

$$
\begin{gathered}
\lg N_{\mathrm{f}}=-(Q+1) \lg \sigma_{\mathrm{a}}+\lg \Phi \\
\Phi=\frac{1}{P\left(1+h^{2}\right)}\left(1-\frac{1}{Q+2}\right)
\end{gathered}
$$

式中, $N_{\mathrm{f}}$ 为构件的预测寿命; $\sigma_{\mathrm{a}}$ 对称加载应力幅。 式(21)为光滑试件理论 $S-N$ 曲线方程。为了获取材 质参数 $Q$ 和 $\Phi$, 本文依据最小二乘法基本原理以及 多元函数求解极值的基本条件, 获得确定材料参数 $Q$ 和 $\Phi$ 的数学模型为

$$
\left\{\begin{array}{c}
Q=\frac{\left(\sum_{i=1}^{j} \lg N_{\mathrm{f}, \mathrm{i}}\right)\left(\sum_{i=1}^{j} \lg \sigma_{\mathrm{a}, \mathrm{i}}\right)-j \sum_{i=1}^{j} \lg N_{\mathrm{f}, \mathrm{i}} \lg \sigma_{\mathrm{a}, \mathrm{i}}}{j \sum_{i=1}^{j}\left(\lg \sigma_{\mathrm{a}, \mathrm{i}}\right)^{2}-\left(\sum_{i=1}^{j} \lg \sigma_{\mathrm{a}, \mathrm{i}}\right)^{2}}-1 \\
\lg \Phi=\frac{1}{j} \sum_{i=1}^{j} \lg N_{\mathrm{f}, \mathrm{i}}+\frac{(Q+1)}{j} \sum_{i=1}^{j} \lg \sigma_{\mathrm{a}, \mathrm{i}}
\end{array}\right.
$$

式中, $j$ 表示试验成组数目; $N_{\mathrm{f}, \mathrm{i}}$ 表示各组试验中 试件中值疲劳寿命; $\sigma_{\mathrm{a}, \mathrm{i}}$ 表示各组试验施载应力幅 值。这里均假定试件的加工方式、表面质量等因素 与国标中的标准试件均相同。至此, 通过式(23)、(24) 以及式(8)可获得材料参数 $Q 、 P$ 以及微塑性循环应 变硬化指数 $n$ 。以下介绍通过非比例加载下的多 轴疲劳试验数据并采用一维黄金分割法获取材料常 数 $g$ 。

由刻画潜在滑移系开启度的旋转因子 $F$ 的具 体表述形式可知

$$
0 \leqslant F \leqslant 1
$$

此外, 微塑性应变作为耗散功过程 ${ }^{[20]}$, 对于一般具 有强化效应的稳定材料而言, 应当满足 Drucker 公
设 ${ }^{[21]}$, 即

$$
\mathrm{d} \sigma \cdot \mathrm{d} e_{\mathrm{p}}>0
$$

这表明微塑性循环应变硬化指数 $n$ 有一定的取值范 围, 即

$$
n>0
$$

疲劳损伤得以不断进行, 归因于塑性应变的不 断累积。非比例多轴高周疲劳较比例加载或单轴加 载具有更短的寿命, 意味着附加强化行为使得材料 的塑性应变累积加快。因此根据上述推演可知, $g$ 参 量亦具有确定的取值范围, 即

$$
-1<g<0
$$

$g$ 取值应使预测寿命 $N_{\mathrm{i}}^{(\mathrm{p})}(g)$ 尽量接近真实寿命, 即

$$
f(g)=\sum_{i=1}^{X}\left[\left(N_{\mathrm{i}}^{(\mathrm{p})}(g)-N_{\mathrm{f}, \mathrm{i}}\right)^{2}\right] \rightarrow \min
$$

式中, $X$ 为任一非比例加载路径下的疲劳试验数据 中取点数目, $X$ 最小取 1 即可识别 $g$ 值, $N_{\mathrm{f}, \mathrm{i}}$ 为疲 劳试验中值寿命, $f(g)$ 表示误差评估函数。易见求 取式(29)的过程实际上是简单的一维优化问题, 可 通过一维黄金分割法 ${ }^{[22]}$ 予以求解, 搜索范围已由式 (28)给出, 计算框图如图 1 所示。应当指出的是, 预测寿命 $N_{\mathrm{i}}^{(\mathrm{p})}(g)$ 很难得到解析解, 可通过数值计算 方法得到，具体的计算方法将在第 3 节说明。



图 1 材料常数 $g$ 搜索寻优计算框图

\section{3 模型的有效性验证}

\section{1 材料参数识别}

笔者选取了飞机制造业常用铝合金 LY12CZ 以 
及 $30 \mathrm{CrMnSiA}$ 钢验证多轴损伤演化模型的可靠性 以及材料参数识别方法的可行性, 两种材料的基本 力学性能参数见表 $1^{[23]}$ 。

\section{表 1 两种材料基本力学性能}

\begin{tabular}{lcccc}
\hline \multicolumn{1}{c}{ 材料 } & $\begin{array}{c}\text { 弹性模量 } \\
E / \mathrm{GPa}\end{array}$ & $\begin{array}{r}\text { 屈服强度 } \\
\sigma_{\mathrm{s}} / \mathrm{MPa}\end{array}$ & $\begin{array}{c}\text { 抗拉强度 } \\
\sigma_{\mathrm{b}} / \mathrm{MPa}\end{array}$ & $\begin{array}{c}\text { 泊松 } \\
\text { 比 } \nu\end{array}$ \\
\hline $\mathrm{LY} 12 \mathrm{CZ}$ & 73 & 400 & 545 & 0.33 \\
$30 \mathrm{CrMnSiA}$ & 203 & 1105 & 1177 & 0.30 \\
\hline
\end{tabular}

文献[23]中，采用的加载方案为利用电液伺服 疲劳试验机 MTS 809 对光滑薄壁圆管试件同时进 行正弦波拉伸和扭转施载, 施载方案如图 2 所示。 此外, 为了获得多组多轴非比例荷载, 文献中通过 改变拉扭方向正弦波载荷的相位角差值, 使得双向 荷载非比例施加, 拉扭荷载瞬时应力值采用公式描 述为

$$
\left\{\begin{array}{l}
\sigma_{x}=\sigma_{\mathrm{a}} \sin (\omega t) \\
\tau_{x y}=\tau_{\mathrm{a}} \sin (\omega t+\psi)
\end{array}\right.
$$

式中, $\sigma_{x}$ 为轴向加载瞬时应力值; $\tau_{x y}$ 为切向加载 瞬时应力值; $\sigma_{\mathrm{a}}$ 轴向加载应力幅值; $\tau_{\mathrm{a}}$ 为切向加载 应力幅值; $\omega$ 为加载频率; $\psi$ 为扭拉相位差值。

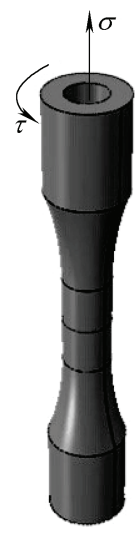

图 2 拉扭薄壁圆管试件

文献中完成两种材料共 88 个疲劳试验, 其中 有效试验 $\mathrm{LY} 12 \mathrm{CZ}$ 共 65 个, $30 \mathrm{CrMnSiA}$ 钢共 19 个。 单轴拉压试验 $\mathrm{LY} 12 \mathrm{CZ}$ 进行了 4 组, 而 $30 \mathrm{CrMnSiA}$ 钢只进行了 4 组纯扭疲劳试验。考虑到材料常数识 别模型式(23)完全基于材料的 $S-N$ 曲线方程, 为了 应用于 $30 \mathrm{CrMnSiA}$ 钢的材料参数识别, 本文依据 von Mises 等效准则将对称纯扭加载应力等效为对 称拉压加载应力, 这种处理是否合适有待后面的进 一步验证。为了获得材料常数 $g$, 本文根据文献中 相位差值为 $90^{\circ}$ 多轴非比例加载路径下的试验数据 并通过一维黄金分割法最佳回归获取了 $g$ 的优化结 果, 最终获得的两种材料的多轴疲劳伤演模型的材 料参数由表 2 给出。
表 2 两种材料多轴疲劳伤演模型材质参数

\begin{tabular}{|c|c|c|c|c|}
\hline 材料 & $\begin{array}{c}\text { 材料常数 } \\
g \\
g\end{array}$ & $\begin{array}{c}\text { 材料常数 } \\
Q \\
Q\end{array}$ & $\begin{array}{c}\text { 材料常数 } \\
P \\
P\end{array}$ & $\begin{array}{l}\text { 微塑性循环 } \\
\text { 硬化指数 } n\end{array}$ \\
\hline LY12CZ & -0.2827 & 6.41 & $2.606 \times 10^{-23}$ & 0.185 \\
\hline $30 \mathrm{CrMnSiA}$ & -0.5188 & 7.07 & $1.249 \times 10^{-28}$ & 0.165 \\
\hline
\end{tabular}

\section{2 模型验证}

由于材料的损伤状态关乎材料后继的承载能 力, 即基于应变等效性假设, 损伤变量 $D$ 会影响材 料演化过程中的本构关系。由前面的推演可知，在 一定的循环次数增量 $\Delta N$ 条件下, 损伤增量 $\Delta D$ 即为

$$
\Delta D=\frac{P(1+g F)^{-\frac{1}{n}} \Delta N}{(Q+1)(1-D)^{Q+1}} \sum_{i=1}^{m}\left(\sigma_{i, \max }^{Q+1}-\sigma_{i, \min }^{Q+1}\right)
$$

考虑到高周疲劳寿命一般较长, 在较小的循环次数 增量条件下，损伤变量一般变化较小。基于此，笔 者采用划分循环次数增量 $\Delta N$ (本文取为 100 周次) 并假设在步长计算范围内材料的本构关系不发生变 化计算拉扭光滑试件的多轴疲劳损伤累积。当累积 的损伤变量 $D$ 到达临界损伤值 $D_{\mathrm{c}}$ (本文取为 1 ) 时, 受损单元失效破坏。另外，从式(31)中可知折算应 力载荷谱峰谷点对损伤累积计算有意义, 中间应力 值意义不大，可将其滤去。根据该计算原理可建立 拉扭光滑试件预测寿命计算框图如图 3 所示。

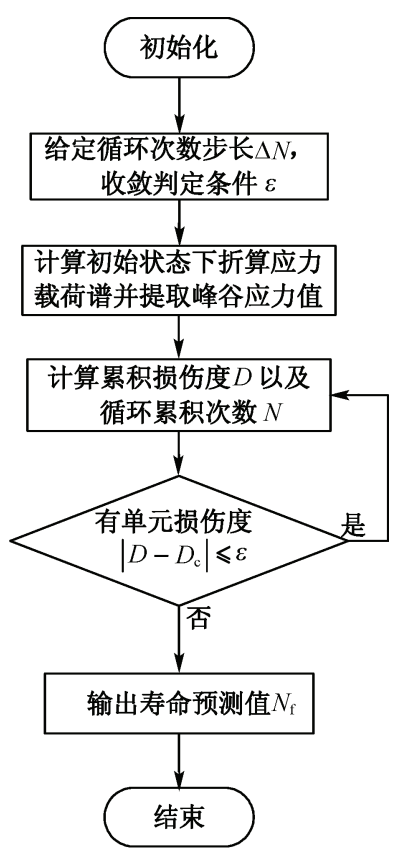

图 3 光滑薄壁圆管试件多轴高周疲劳寿命计算框图

根据图 3 的计算原理并基于 Fortran 语言编写了 UMAT 子程序, 将其嵌入 ABAQUS 主程序中, 实 现试件的损伤追踪以及寿命预测。采用 8 节点减缩 
积分单元对试件进行网格划分。考虑到试件在扭拉 加载下存在一定的应力梯度, 因此划分的单元尺寸 关乎试件的寿命预测精度, 本文参考文献[12]的网 格划分尺度, 将试验段的单元尺寸划为 $0.5 \mathrm{~mm}$ 。应 当指出的是, 本文在上述的参数识别以及寿命预测 中, 均忽略了疲劳裂纹扩展寿命, 这主要是由于对 于无类裂纹缺陷试件的高周疲劳裂纹形成寿命占据 全寿命的主要部分, 甚至达到 $90 \%$ 以上 $^{[24]}$, 这种忽 略是可取的。

图 4 给出了表 3 中 $\mathrm{LY} 12 \mathrm{CZ}$ 铝合金 7 号试件当 循环次数到达 30000 周次时的损伤场分布云图, 由 云图可知, 损伤主要发生在试件的试验段。此外, 图 5 还给出了该试件中临界损伤单元的损伤累积历 程曲线, 由曲线可知, 在循环加载初期, 损伤累积 基本符合 Miner 线性累积准则, 但当后期直至试件 断裂失效, 损伤累积非线性较强。

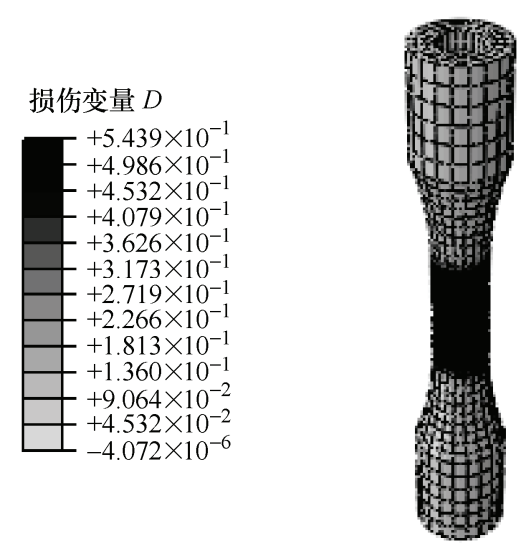

图 4 LY12CZ-7 号试件循环 30000 周次损伤分布云图

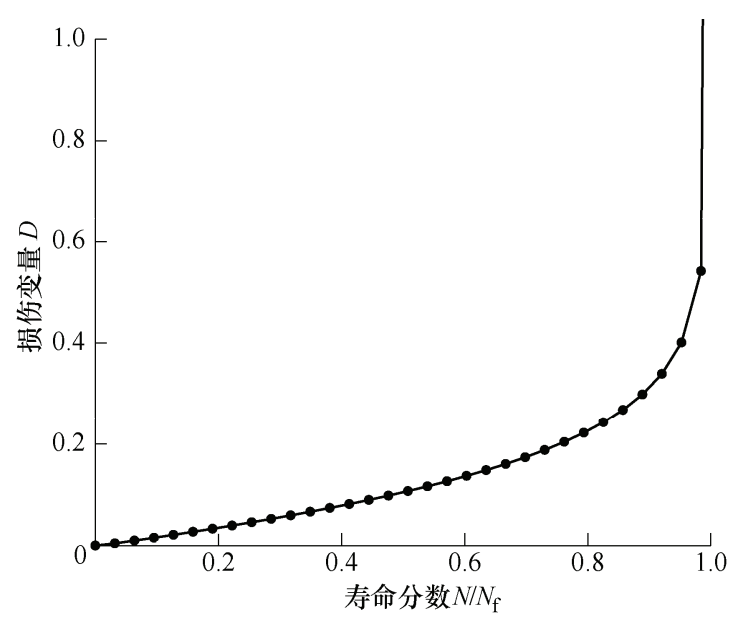

图 5 LY12CZ-7 号试件临界损伤单元损伤历程曲线

本文模型对两种材料在不同加载路径下的多 轴疲劳寿命预测结果见表 3 和表 4 。对比表中的数 据可知, 本文模型对两种不同类型材料的多轴高周 疲劳寿命的预测效果均在 3 倍误差带以内, 与试验 吻合较好。
表 $3 \mathrm{LY12CZ}$ 多轴高周疲劳寿命预测值及试验值对比

\begin{tabular}{cccccc}
\hline 序号 & $\begin{array}{c}\text { 轴向应力幅 } \\
\sigma_{\mathrm{a}} / \mathrm{MPa}\end{array}$ & $\begin{array}{c}\text { 切向应力幅 } \\
\tau_{\mathrm{a}} / \mathrm{MPa}\end{array}$ & $\begin{array}{c}\text { 相位差值 } \\
\psi /\left(^{\mathrm{O}}\right)\end{array}$ & $\begin{array}{c}\text { 中值疲劳 } \\
\text { 寿命 } N_{\mathrm{f}}\end{array}$ & $\begin{array}{c}\text { 预测寿命 } \\
N_{\mathrm{f}}^{(\mathrm{p})}\end{array}$ \\
\hline 1 & 126.491 & 91.571 & 0 & 482666 & 211200 \\
2 & 189.737 & 137.356 & 0 & 23003 & 12200 \\
3 & 158.114 & 111.803 & 0 & 76451 & 50800 \\
4 & 126.491 & 95.507 & 30 & 420261 & 175300 \\
5 & 158.114 & 119.384 & 30 & 63584 & 33400 \\
6 & 126.491 & 100.000 & 45 & 275527 & 155700 \\
7 & 158.114 & 125.000 & 45 & 57004 & 30200 \\
8 & 126.491 & 105.193 & 60 & 231348 & 152800 \\
9 & 158.114 & 131.491 & 60 & 30893 & 29600 \\
10 & 200.000 & 115.470 & 90 & 14296 & 39300 \\
11 & 250.000 & 144.340 & 90 & 4634 & 7800 \\
12 & 126.491 & 111.289 & 90 & 66940 & 190100 \\
13 & 158.114 & 139.111 & 90 & 15459 & 36800 \\
$14 *$ & 200.000 & 100.000 & 90 & 37789 & 38300 \\
$15 *$ & 250.000 & 125.000 & 90 & 6811 & 7600 \\
\hline
\end{tabular}

注: *为识别材料常数 $g$ 的加载路径。

表 $430 \mathrm{CrMnSiA}$ 多轴高周疲劳寿命预测值及试验值对比

\begin{tabular}{cccccc}
\hline 序号 & $\begin{array}{c}\text { 轴向应力幅 } \\
\sigma_{\mathrm{a}} / \mathrm{MPa}\end{array}$ & $\begin{array}{c}\text { 切向应力幅 } \\
\tau_{\mathrm{a}} / \mathrm{MPa}\end{array}$ & $\begin{array}{c}\text { 相位差值 } \\
\psi /\left(^{\circ}\right)\end{array}$ & $\begin{array}{c}\text { 中值疲劳 } \\
\text { 寿命 } N_{\mathrm{f}}\end{array}$ & $\begin{array}{c}\text { 预测寿命 } \\
N_{\mathrm{f}}^{(\mathrm{p})}\end{array}$ \\
\hline 1 & 368.521 & 266.7830 & 0 & 104143 & 284400 \\
2 & 368.521 & 291.3415 & 45 & 92309 & 94200 \\
$3 *$ & 368.521 & 324.2300 & 90 & 71822 & 61500 \\
$4 *$ & 268.100 & 324.2300 & 90 & 105211 & 114000 \\
\hline
\end{tabular}

注: $*$ 为识别材料常数 $g$ 的加载路径。

为了更好地比较预测效果, 绘制了本文预测模 型、McDiarmid 和 Papadopoulos 寿命预测模型对两 种材料在多轴加载下的预测效果对比图, 如图 6 和 图 7 所示, 其中 McDiarmid 和 Papadopoulos 寿命预 测值均来自文献[23]。

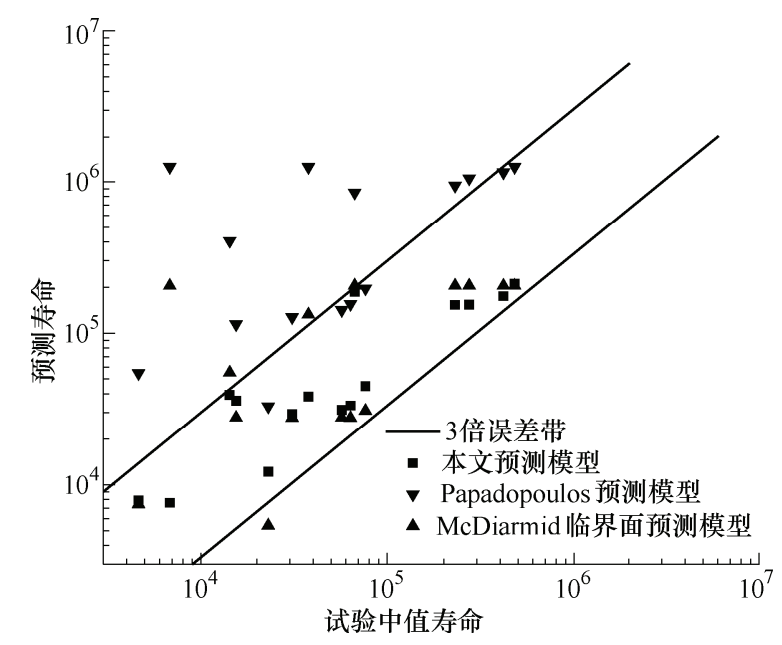

图 $6 \mathrm{LY} 12 \mathrm{CZ}$ 铝合金模型预测效果对比图

由图 6 和图 7 可知, 本文模型对两种不同类型 材料的寿命预测数据点均位于 3 倍误差带之内, 与 试验吻合较好, 而 McDiarmid 和 Papadopoulos 预测 


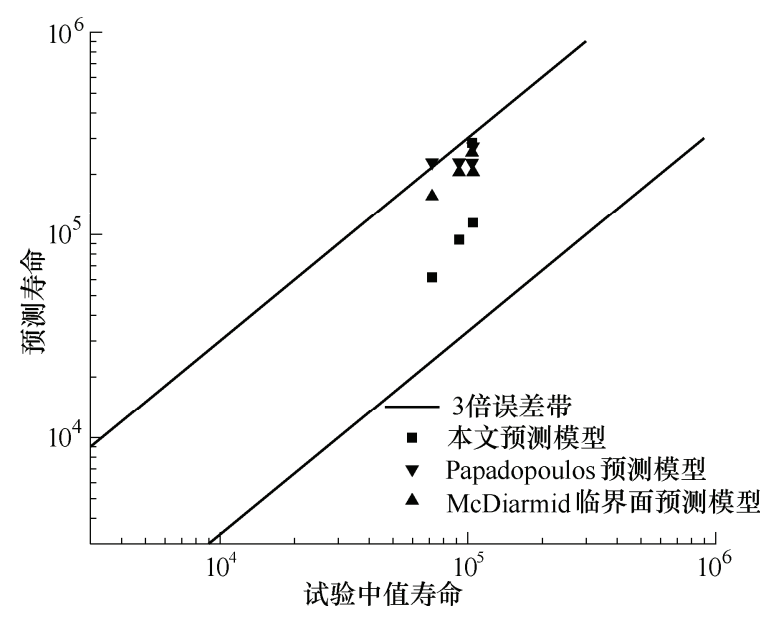

图 $730 \mathrm{CrMnSiA}$ 钢模型预测效果对比图

模型对铝合金 LY12CZ 的预测效果分别仅有 $40 \%$ 和 $80 \%$ 数据点位于 3 倍误差带之内, 对 $30 \mathrm{CrMnSiA}$ 钢 的预测效果 McDiarmid 预测模型的预测效果均位于 3 倍误差带以内, 而 Papadopoulos 预测模型有部分 数据点远离 3 倍误差带, 对比分析表明本文模型更 适合于多轴高周疲劳寿命预测。

需要指出的是, 本文采用了拉扭光滑试件在无 平均应力保持作用下的多轴疲劳试验数据对所建模 型进行验证, 对于缺口试件或具有平均应力工况是 否适用, 还需要进一步验证。

\section{4 结论}

(1) 基于 LEMAITRE 一般意义上损伤演化方 程, 并结合高周疲劳损伤特点, 考虑到多轴高周疲 劳损伤的非比例附加强化效应, 建立了计及非比例 附加强化效应的多轴高周疲劳损伤演化模型, 并对 伤演模型的材料常数识别方法进行了阐述。通过铝合 金 LY12CZ 和 30CrMnSiA 钢两种不同类型材料已有 的高周㽻劳试验数据获得了伤演模型中的材料参数。

(2) 将计及非比例附加强化的多轴高周疲劳伤 演模型编程嵌入到 ABAQUS 有限元软件 UMAT 子 程序中, 实现了对铝合金 LY12CZ 和 $30 \mathrm{CrMnSiA}$ 钢 试件在多种非比例加载路径下的临界损伤单元的损 伤追踪以及寿命预测。计算结果表明, 计算寿命与 试验结果误差均在 3 倍误差带以内, 与试验吻合较 好, 明显优于现有的预测模型, 本文模型更适合于 多轴高周疲劳寿命预测。

(3) 对于具有平均应力保持工况或缺口试样的 多轴荷载, 模型的适用性有待试验的进一步论证。

\section{参 考 文 献}

[1] 谭文锋. 损伤力学方法预估构件的疲劳裂纹形成寿命 [D]. 秦皇岛: 燕山大学, 2009 .

TAN Wenfeng. Predicting fatigue crack initiation lives of components by using damage mechanics[D]. Qinhuangdao: Yanshan University, 2009

[2] GOUGH H J. Crystalline structure in relation to fatigue of metals especially fatigue[C]//Proceedings of the American Society for Testing and Materials 33. Philadelphia, PA: American Society for Testing and Materials, 1933: 3-22.

[3] MCDIARMID D L. A general criterion for high cycle multiaxial fatigue failure[J]. Fatigue Fract. Engug. Mater. Struct., 1991, 14: 429-453.

[4] PAPADOPOULOS I V. Long life fatigue under multiaxial loading[J]. Int. J. Fatigue， 2001， 23: 839-849.

[5] CROSSLAND B. Effect of large hydrostatic pressures on the torsional fatigue strength of an alloy steel[J]. Institution of Mechanical Engineers, 1956: 184-194.

[6] SINES G. Behavior of metals under complex static and alternating stresses[J]. Matal. Fatigue, 1959: 145-169.

[7] WANG Yingyu , YAO Weixing. Evaluation and comparison of several multiaxial fatigue criteria[J]. Int. J. Fatigue, 2004, 26: 17-25.

[8] 金丹, 王巍, 田大将, 等. 非比例载荷下缺口件㽻劳寿 命有限元分析[J]. 机械工程学报, 2014，50(2): 25-29. JIN Dan, WANG Wei, TIAN Dajiang, et al. Finite element analysis of fatigue life for notched specimen under nonproportional loading[J]. Journal of Mechanical Engineering, 2014, 50(2): 25-29.

[9] 张成成, 姚卫星. 一种新的多轴高周疲劳寿命预测模型 [J]. 力学学报, 2010, 42(6): 1225-1230.

ZHANG Chengcheng, YAO Weixing. A new model for life prediction of multiaxial high-cycle fatigue[J]. Chinese Journal of Theoretical and Applied Mechanics, 2010, 42(6): 1225-1230.

[10] 蔡能, 尚德广. 高温多轴疲劳损伤与寿命预测研究进展 [J]. 机械强度, 2004，26(5): 576-581。

CAI Neng, SHANG Deguang. Advances in research of multiaxial fatigue damage and life prediction at high temperature[J]. Journal of Mechanical Strength, 2004, 26(5): 576-581.

[11] 郑占光, 蔡敢为, 李兆军, 等. 基于损伤力学阐释 Manson-Coffin 低周疲劳模型 $[\mathrm{J}]$. 中国机械工程, 2011, 22(7): 812-814.

ZHENG Zhanguang, CAI Ganwei, LI Zhaojun, et al. Interpretation of Manson-Coffin model of low cycle fatigue based on damage mechanics[J]. China Mechanical Engineering, 2011, 22(7): 812-814.

[12] 朱正宇, 何国求, 陈成澍, 等. 多轴非比例加载高周疲 劳研究进展 [J]. 同济大学学报, 2006, 34(9): 1221-1225. ZHU Zhengyu, HE Guoqiu, CHEN Chengshu, et al. Recent advances of multiaxial high cycle fatigue under 
nonproportional loading[J]. Journal of Tongjing University, 2006, 34(9): 1221-1225.

[13] LEMAITRE J. A course on damage mechanics[M]. Berlin: Spring-Verlag, 1992.

[14] 余寿文, 冯西桥. 损伤力学[M]. 北京: 清华大学出版 社, 1997.

YU Shouwen, FENG Xiqiao. Damage mechanics[M]. Beijing: Tsinghua University Press, 1997.

[15] 刘新东, 郝际平. 连续介质损伤力学 [M]. 北京: 国防 工业出版社, 2011.

LIU Xindong, HAO Jiping. Continuum damage mechanics[M]. Beijing: National Defence Industry Press, 2011.

[16] 杨锋平, 孙勤, 罗金恒, 等. 一个高周疲劳损伤演化修 正模型[J]. 力学学报, 2012, 44(1): 140-146.

YANG Fengping, SUN Qin, LUO Jinheng, et al. A corrected damage law for high cycle fatigue[J]. Chinese Journal of Theoretical and Applied Mechanics, 2012, 44(1): $140-146$.

[17] 丁新星. 基于 ANSYS 的损伤力学-有效应力法预估构 件寿命[D]. 秦皇岛: 燕山大学, 2011 .

DING Xinxing. The actual stress method based on damage mechanics to predict life on ANSYS platform[D]. Qinghuangdao: Yanshan University, 2011.

[18] 尚德广, 王德俊. 多轴疲劳强度 $[\mathrm{M}]$. 北京: 科学出版 社, 2007.

SHANG Deguang, WANG Dejun. Multiaxial fatigue strength[M]. Beijing: Science Press, 2007.

[19] 钟炜辉. 钢结构的高周疲劳损伤有限元分析模型研究 [D]. 西安：西安建筑科技大学，2005.

ZHONG Weihui. Studies on finite element analysis model of high cycle fatigue damage for steel structure[D]. Xi'an: Xi'an University of Architecture and Technology,
2005.

[20] 杨晓华, 李年. $2 \mathrm{Cr} 13$ 钢高周疲劳微塑性损伤特性研究 [J]. 金属热处理学报，1999，20(2)：31-36.

YANG Xiaohua , LI Nian. Characterization of micrplasticity damage developed during high cycle fatigue in $2 \mathrm{Cr} 13$ steel[J]. Transactions of Metal Heat Treatment, 1999, 20(2): 31-36.

[21] 尚福林, 王子昆. 塑性力学基础[M]. 西安: 西安交通 大学出版社, 2011.

SHANG Fulin, WANG Zikun. Foundations of plasticity mechanics[M]. Xi'an: Xi'an Jiaotong University Press, 2011.

[22] 孙靖民, 梁迎春. 机械优化设计[M]. 北京：机械工业 出版社, 2006.

SUN Jingmin , LIANG Yingchun. Mechanical optimization design[M]. Beijing: China Machine Press, 2006.

[23] 张成成. 复杂应力场下结构高周疲劳寿命分析[D]. 南 京: 南京航空航天大学, 2010.

ZHANG Chengcheng. Fatigue life prediction of structure in HCF region under complex stress field[D]. Nanjing: Nanjing University of Aeronautics and Astronautics, 2010.

[24] 李臻. 低碳低合金钢的变幅疲劳寿命估算 $[\mathrm{J}]$. 压力容 器, 2006, 7(23): 10-14.

LI Zhen. Fatigue life prediction of low carbon low alloy steel under variable amplitude loading[J]. Pressure Vessel Technology, 2006, 7(23): 10-14.

作者简介: 彭艳(通信作者), 男, 1972 年出生, 博士, 教授, 博士研究 生导师。主要研究方向为金属板带轧制技术。

E-mail: pengyan@ysu.edu.cn

李浩然, 男, 1990 年出生。主要研究方向为材料损伤。

E-mail:948109512@qq.com 